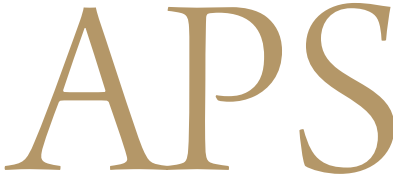

Archives of Plastic Surgery

\title{
L-Shaped Columellar Strut in East Asian Nasal Tip Plasty
}

\author{
Eun-Sang Dhong ${ }^{1}$, Yeon-Jun Kim${ }^{1}$, Man Koon Suh ${ }^{2}$ \\ ${ }^{1}$ Department of Plastic and Reconstructive Surgery, Korea University College of Medicine, Seoul; ${ }^{2}$ WW Plastic Surgery Center, Seoul, Korea
}

Background Nasal tip support is an essential consideration for rhinoplasty in East Asians. There are many techniques to improve tip projection, and among them, the columellar strut is the most popular technique. However, the conventional design is less supportive for rotating the tip. The amount of harvestable septal cartilage is relatively small in East Asians. For an optimal outcome, we propose an L-shaped design for applying the columellar strut.

Methods To evaluate the anthropometric outcomes, the change in nasal tip projection and the columella-labial angle were analyzed by comparing preoperative and postoperative photographs. The anthropometric study group consisted of 25 patients who underwent the same operative technique of an L-shaped strut graft using septal cartilage and were followed up for more than 9 months.

Results There were statistically significant differences between the preoperative and postoperative values in the nasal tip projection ratio and columella-labial angle. We did not observe any complications directly related to the L-shaped columellar strut in the anthropometric study group.

Conclusions The L-shaped columellar strut has advantages not only in the controlling of tip projection and rotation, but in that it needs a smaller amount of cartilage compared to the conventional septal extension graft. It can therefore be an alternative technique for nasal tip plasty when there is an insufficient amount of harvestable septal cartilage.

Keywords Rhinoplasty / Nasal septum / Treatment outcome
Correspondence: Man Koon Suh JW Plastic Surgery Center, Samsin Building, 836 Nonhyeon-ro, Gangnam-gu, Seoul 135-893, Korea Tel: $+82-2-541-5114$ Fax: +82-2-541-5112 E-mail:smankoon@hanmail.net

No potential conflict of interest relevant to this article was reported.

Received: 7 May 2013 • Revised: 29 Jul 2013 • Accepted: 30 Jul 2013

pISSN: 2234-6163 • elSSN: 2234-6171 • http://dx.doi.org/10.5999/aps.2013.40.5.616 • Arch Plast Surg 2013;40:616-620

\section{INTRODUCTION}

Nasal tip support is an important consideration for Asian rhinoplasty. A number of soft tissue components and cartilaginous relationships support the nasal tip. The attachments that connect the upper lateral cartilages and the lower lateral cartilages (LLCs), the lateral crura of the LLCs and the pyriform aperture, the paired domes of the LLCs, the medial crura of the LLCs and the nasal septum, and that of Pitanguy's dermocartilaginous ligament all play important roles [1].
Any dissection of the above structures may damage the support of the nasal tip. Surgical procedures such as transfixation incisions, cephalic trim, intercartilaginous incisions, and medial division of the LLCs disrupt the support and cause changes in the tip position. Therefore, reinforcement of the nasal tip is required in most cases in such procedures. However, most East Asian patients want their tips to be more projected, even if the above procedures are not performed.

There are many techniques for improving tip projection. The columellar strut is one of the most popular techniques. How- 
ever, the columellar strut has some disadvantages [2]. Nasal tip position, including projection and rotation, is less predictable with a fixed or floating strut as opposed to a caudal septal extension graft. There are many surgeons who are optimistic about using a septal extension graft to gain the desired nasal tip projection and rotation at the same time. However, this graft often results in stiffness of the nasal tip [3]. Futhermore, not all patients have enough septal cartilage for proper extension. The amount of septal cartilage harvested is relatively small in Asians, and some portion of the harvest should be left for other grafts in many cases. In addition, in secondary cases, the septal cartilage may have been used before.

For an optimal outcome, we propose a design of an L-shaped columellar strut, which requires a smaller amount of cartilage and serves as a powerful tool for controlling tip projection and rotation.

\section{METHODS}

\section{Study subjects}

The cases of two surgeons (first and third authors) were summarized for this study. All of the patients who underwent the L- shaped columellar strut graft using septal cartilage in this study were primary rhinoplasty cases with insufficient cartilage for tip plasty. The anthropometric study group was chosen by selecting 25 patients who were observed for more than 9 months postoperatively (Table 1). The average follow-up period was 12.3 months. 20 patients wanted to increase the tip projection solely, while 5 patients wanted to rotate the tip cephalically at the same time.

\section{Surgical technique}

After uncovering the skin envelope, subperichondrial dissection of the septum was continued between the medial crura of the LLCs until the caudal septal cartilage was visualized. The septal cartilage was harvested, producing an L-shaped strut of more than $10 \mathrm{~mm}$. Less than $2 \times 1.5 \mathrm{~cm}^{2}$ of the area was harvested from the septal cartilage. The harvest was carved into an L-shaped strut (Fig. 1). The horizontal portion of the strut was fixed to the caudal septum with more than two sutures (Fig. 2A). The vertical portion of the strut was then placed between the LLCs (Fig. 2B). Then, the LLCs were advanced as needed (caudally, cephalically, or anteriorly), and they were temporarily fixed to the graft using a straight needle. The permanent fixations were made after confirming that the tip and columella were appro-

\section{Table 1. Patients' characteristics and anthropometric analysis}

\begin{tabular}{|c|c|c|c|c|c|c|}
\hline Case & Gender/Age (yr) & Main concern & Pre-NTP ratio & Post-NTP ratio & Pre-CLA & Post-CLA \\
\hline 1 & $M / 23$ & Tip projection & 0.73 & 0.96 & 84.5 & 95.4 \\
\hline 2 & $F / 29$ & Tip projection & 0.92 & 1.07 & 77.6 & 91.4 \\
\hline 3 & $F / 28$ & Tip projection & 0.95 & 1.19 & 97.1 & 112.2 \\
\hline 4 & $F / 25$ & Tip projection & 0.86 & 1.18 & 99.1 & 107.6 \\
\hline 5 & $\mathrm{M} / 30$ & Tip projection & 0.86 & 1.18 & 90.6 & 94.0 \\
\hline 6 & $M / 31$ & Tip rotation & 0.81 & 1.12 & 58.2 & 81.7 \\
\hline 7 & $F / 22$ & Tip projection & 0.92 & 1.02 & 87.0 & 88.2 \\
\hline 8 & $F / 25$ & Tip rotation & 0.93 & 1.24 & 77.6 & 100.7 \\
\hline 9 & $F / 31$ & Tip projection & 0.92 & 1.12 & 92.5 & 98.3 \\
\hline 10 & $F / 26$ & Tip projection & 0.85 & 1.24 & 94.3 & 99.2 \\
\hline 11 & $\mathrm{~F} / 27$ & Tip projection & 0.88 & 1.26 & 90.9 & 91.3 \\
\hline 12 & $F / 22$ & Tip projection & 0.95 & 1.23 & 90.5 & 100.4 \\
\hline 13 & $F / 29$ & Tip projection & 0.89 & 1.14 & 83.8 & 92.7 \\
\hline 14 & $M / 25$ & Tip projection & 0.79 & 1.11 & 79.7 & 83.3 \\
\hline 15 & $F / 34$ & Tip rotation & 0.85 & 1.05 & 65.1 & 86.8 \\
\hline 16 & $F / 26$ & Tip projection & 0.84 & 1.20 & 93.8 & 102.5 \\
\hline 17 & $F / 32$ & Tip rotation & 0.71 & 0.94 & 72.6 & 94.5 \\
\hline 18 & $F / 20$ & Tip projection & 0.86 & 1.02 & 79.5 & 84.7 \\
\hline 19 & $F / 28$ & Tip projection & 0.82 & 1.17 & 94.7 & 101.2 \\
\hline 20 & $\mathrm{M} / 25$ & Tip rotation & 0.87 & 1.17 & 61.6 & 81.1 \\
\hline 21 & $F / 26$ & Tip projection & 0.74 & 0.99 & 81.6 & 93.4 \\
\hline 22 & $F / 29$ & Tip projection & 0.89 & 1.16 & 86.4 & 97.7 \\
\hline 23 & $F / 31$ & Tip projection & 0.82 & 1.12 & 87.2 & 98.5 \\
\hline 24 & $M / 24$ & Tip projection & 0.92 & 1.06 & 93.8 & 99.2 \\
\hline 25 & $F / 32$ & Tip projection & 0.76 & 1.08 & 85.5 & 95.6 \\
\hline Mean & & & $0.85 \pm 0.07$ & $1.12 \pm 0.09^{\mathrm{a})}$ & $84.2 \pm 10.8$ & $94.9 \pm 7.7^{\mathrm{b}}$ \\
\hline
\end{tabular}

NTP, nasal tip projection; CLA, columella-labial angle.

$\left.{ }^{a, b}\right)$ There were statistically significant differences between the preoperative and postoperative values of the nasal tip projection ratio and columella-labial angle $(P<0.05)$. 
priately reshaped. The domal segment of the alar cartilage and vertical portion of the strut were sutured at several points for tip projection and rotation (Fig. 2C, D). Any excess of the graft was trimmed.

\section{Anthropometric analysis and statistical analysis}

To evaluate the outcomes, nasal tip projection and the columellalabial angle were analyzed by photography with a lateral view, and by comparing preoperative and postoperative photographs. The postoperative photographs that were taken at least 9 months after the operation were used for the analysis. The columella-labial angle was determined by drawing a line from the subnasale to the labrale superius. The angle between this line and the line from the subnasale to the most inferior columella is considered the columella-labial angle. Nasal tip projection was measured from the nasolabial angle to the point of intersection with a line

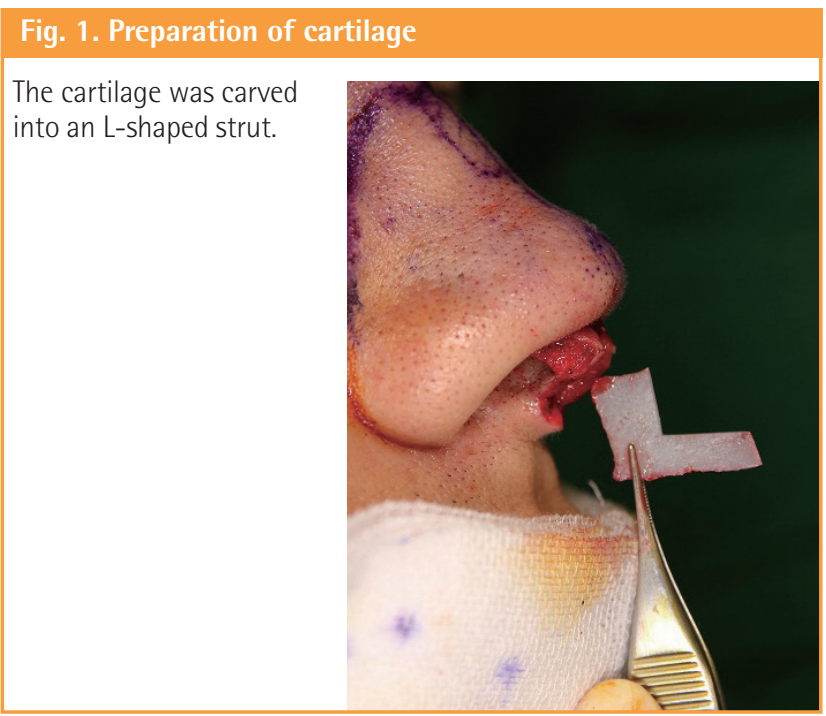

from the nasion along the dorsum [4]. The distance between the lateral mouth corner (commissure) and the upper margin of Cupid's bow (labrale superius) were measured for the standard. Each photograph was restored using Adobe Illustrator software, and the nasal tip projection was measured using the "line segment tool” from Adobe Illustrator (Adobe Systems Inc., San Jose, CA, USA).

Nasal tip projection was standardized as the measurement of the distance between the commissure and the labrale superius, and the nasal tip projection ratio was determined as the nasal tip projection/distance between the commissure and the labrale superius (Fig. 3) [5]. The results were analyzed with the paired t-test and Wilcoxon signed rank test, and a P-value of less than 0.05 was considered statistically significant.

Fig. 3. Nasal tip projection and columella-labial angle measurement

CLA, columella-labial angle $\mathrm{NL}$, indicates nasal length; NTP, nasal tip projection.

(A) The horizontal portion of the strut was sutured to the caudal septum. (B) The vertical portion of the strut was placed in between the lower alar cartilage. (C) The domal segment of the alar cartilage and the strut were sutured at two points. (D) Schematic diagram of the L-shaped columellar strut technique.
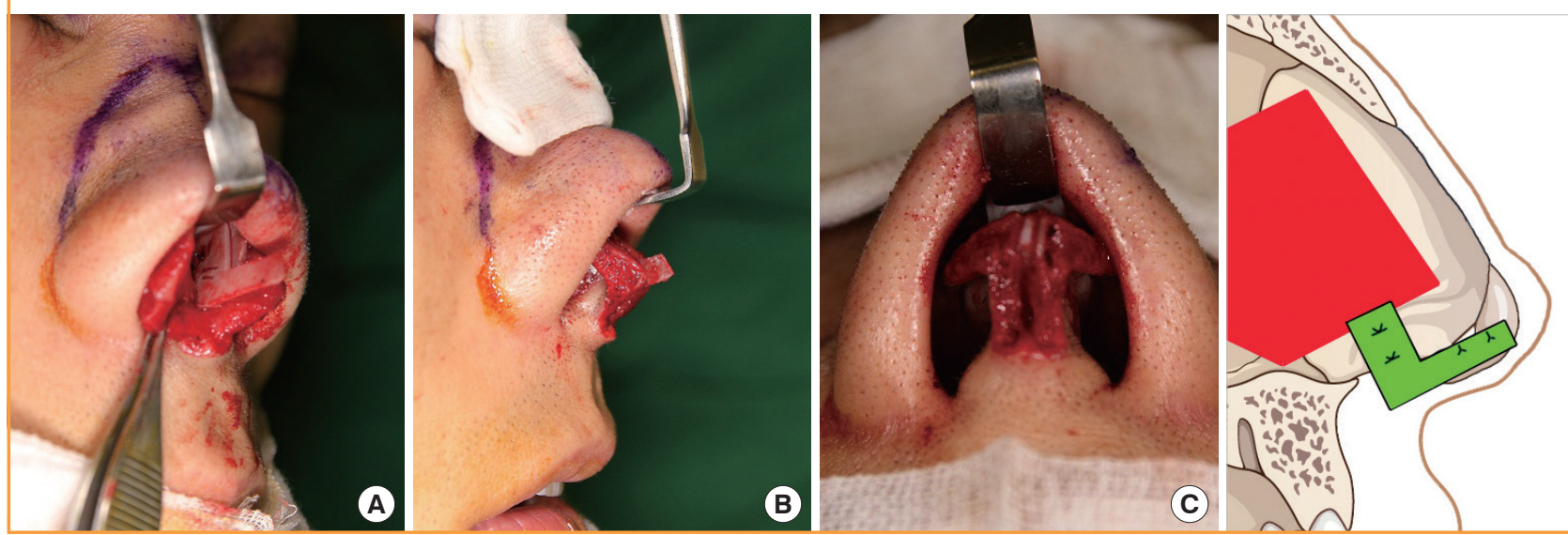


\section{RESULTS}

Table 1 shows the changes in the nasal tip projection ratio and the columella-labial angle after the operation. There were statistically significant differences between the pre- and postoperative values of the nasal tip projection ratio and columella-labial angle $(\mathrm{P}<0.05)$. Preoperatively, the average nasal tip projection ratio (nasal tip projection/distance between the commissure and the labrale superius) was 0.85 (0.07) (mean [SD]), and $1.12(0.09)$ postoperatively. The average preoperative and postoperative columella-labial angle were 84.2 (10.8) (mean [SD]) and 94.9 (7.7) respectively. The procedure with the L-shaped columellar strut therefore increased the nasal tip projection by an average of $32 \%$. We observed that the columella-labial angle was also increased by an average of $13 \%$. We did not observe any complications directly related to the L-shaped columellar strut.

\section{Representative cases}

\section{Case 1}

A 22-year-old female patient presented with a small nose. She had insufficient septal cartilage for a septal extension graft. A dorsal silicone implant (nasion to supratip area) and tip plasty with an L-shaped columellar strut graft and shield graft were performed (Fig. 4).

\section{Case 2}

A 25-year-old female patient with a history of submucous septal

\section{Fig. 4. Case 1. A 22-year-old patient}

(A) Preoperative lateral view. (B) One-year postoperative lateral view.
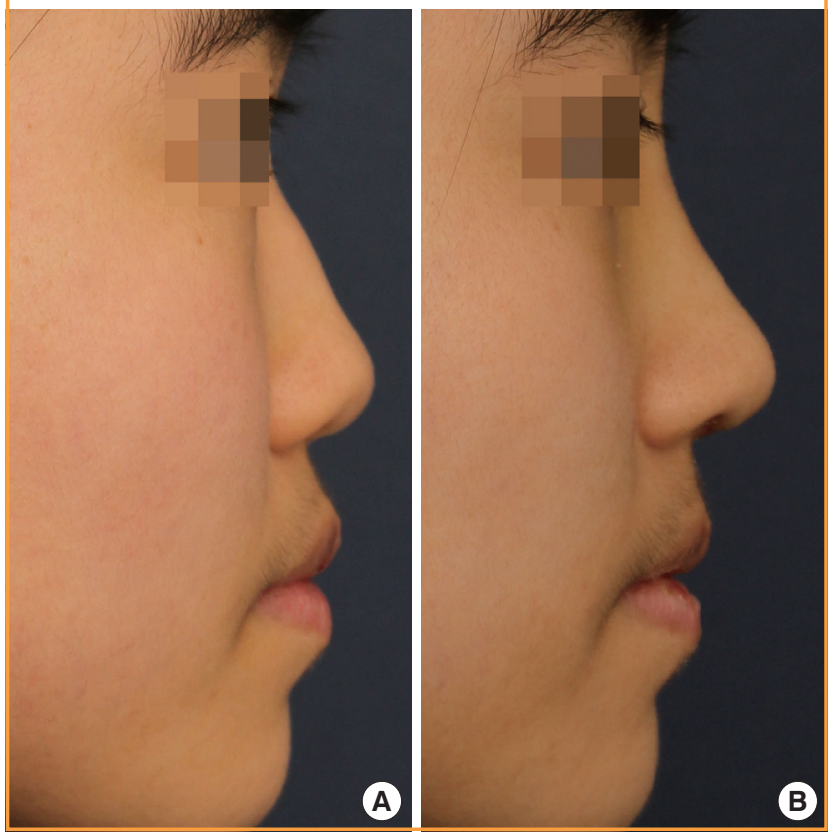

resection presented with a long nose and an acute columellalabial angle. She wanted tip projection with cephalic rotation. An L-shaped columellar strut graft was used for tip plasty using the same methods previously mentioned (Fig. 5).

\section{DISCUSSION}

The nasal tip of ethnic East Asians, compared with that of Caucasians, has round or bulbous shaped features, a lower height, and a wider base. This is due to a longer distance between the alar cartilages, a weaker structure of the alar cartilages, and thicker and more fibrous dermis and subcutaneous tissue in the nasal tip [1].

Various techniques have been used to reposition the nasal tip and the columella. Among these, the columellar strut provides satisfactory structural tip support to gain projection and rotation. It is also used to equalize and maintain the shape of the medial crura, to change the degree of columellar show, and to refine the infratip columella-lobule region. When used properly and with appropriate surgical maneuvers, the results are predictable and consistent $[6,7]$.

However, the limitations of the columellar strut and its failure to control the projection, rotation, and shape of the nasal tip have been discussed by several authors [2,8-10]. Columellar struts are subject to both resorption and displacement. In addition, columellar struts do not prevent cephalic rotation of the tip-lobule complex and therefore offer no control of the length

\section{Fig. 5. Case 2. A 25-year-old patient}

(A) Preoperative lateral view. (B) Ten-month postoperative lateral view.
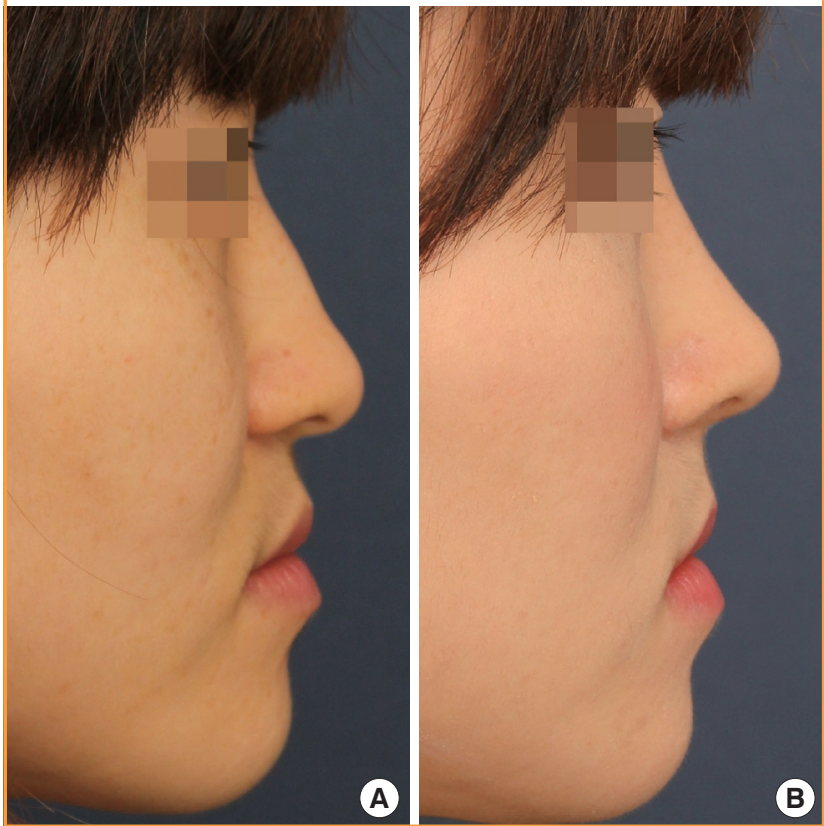
unless attached to the caudal septum. Thus, postoperative tip drooping is commonly found with the columellar strut. Since the columellar strut is not attached to the caudal septum, as it is located primarily between the alar cartilages, it lies only on the anterior-posterior axis [11]. Therefore, it is inadequate for rotating the tip or improving the nasal angle.

In 1997, Byrd et al. [2] introduced septal extension grafts as a method of controlling tip projection and shaping in a series of patients with weak lower lateral cartilages. The grafts were described in three forms: paired spreader grafts, paired batten grafts, and direct extension grafts. Nasal tip stiffness and thickening of the septum in the nasal valve area are the major drawbacks of paired septal extension grafts [3]. The direct extension graft shows some similarities to the L-shaped columellar strut. However, as in the columellar strut, its fixation is uni-dimensional as it is placed on the cephalic-caudal axis only. Moreover, extension grafts may require a large amount of cartilage for adequate strength, often requiring more than what is available. Conchal cartilage is commonly used as additional graft material and costal cartilage grafting may be needed in some cases [12].

We described a modified technique that differs from the columellar strut or the septal extension graft. There are several advantages to using the L-shaped columellar strut. As the Lshaped columellar strut is fixed to the caudal septum, with its vertical portion positioned between the medial crura, a droopy tip is less common than with the floating columellar strut. Its fixation is not too strong, which allows the nasal tip to feel smoother and more flexible than with the fixed columellar strut or the septal extension graft. In addition, it enables surgeons to put the tip exactly where they desire in terms of projection and rotation. Moreover, as the L-shaped columellar strut is secured to the base of the columella, a retracted columellar base can be corrected simultaneously with nasal tip projection.

The septal cartilage is often so hypoplastic in Asians that its amount is almost always insufficient [13]. Furthermore, the septa have often been previously resected in most secondary rhinoplasty procedures. The L-shaped columellar strut uses a smaller amount of cartilage than the septal extension graft. Thus, it allows us to use the cartilage remnant as additional tip graft material like an onlay graft or shield graft.

Limitations of the study include a small case size, absence of a control group and short-term follow-up. For these reasons, a study with a longer duration and a larger sample size is currently in progress to confirm the long-term stability of the L-shaped columellar strut graft. Efforts are currently being made to collect and analyze more photographs and data.

\section{REFERENCES}

1. Han SK, Lee DG, Kim JB, et al. An anatomic study of nasal tip supporting structures. Ann Plast Surg 2004;52:134-9.

2. Byrd HS, Andochick S, Copit S, et al. Septal extension grafts: a method of controlling tip projection shape. Plast Reconstr Surg 1997;100:999-1010.

3. Seyhan A, Ozden S, Ozaslan U, et al. A simplified use of septal extension graft to control nasal tip location. Aesthetic Plast Surg 2007;31:506-11.

4. Wang JH, Jang YJ, Park SK, et al. Measurement of aesthetic proportions in the profile view of Koreans. Ann Plast Surg 2009;62:109-13.

5. Suh MK, Ahn ES, Kim HR, et al. A 2-year follow-up of irradiated homologous costal cartilage used as a septal extension graft for the correction of contracted nose in Asians. Ann Plast Surg 2013;71:45-9.

6. Rohrich RJ, Hoxworth RE, Kurkjian TJ. The role of the columellar strut in rhinoplasty: indications and rationale. Plast Reconstr Surg 2012;129:118e-125e.

7. Ghavami A, Janis JE, Acikel C, et al. Tip shaping in primary rhinoplasty: an algorithmic approach. Plast Reconstr Surg 2008;122:1229-41.

8. Adams WP Jr, Rohrich RJ, Hollier LH, et al. Anatomic basis and clinical implications for nasal tip support in open versus closed rhinoplasty. Plast Reconstr Surg 1999;103:255-61.

9. Petroff MA, McCollough EG, Hom D, et al. Nasal tip projection. Quantitative changes following rhinoplasty. Arch Otolaryngol Head Neck Surg 1991;117:783-8.

10. Hubbard TJ. Exploiting the septum for maximal tip control. Ann Plast Surg 2000;44:173-80.

11. Kim JS, Han KH, Choi TH, et al. Correction of the nasal tip and columella in Koreans by a complete septal extension graft using an extensive harvesting technique. J Plast Reconstr Aesthet Surg 2007;60:163-70.

12. Kang JG, Ryu J. Nasal tip surgery using a modified septal extension graft by means of extended marginal incision. Plast Reconstr Surg 2009; 123:343-52.

13. Han K, Kim J, Son D, et al. How to harvest the maximal amount of conchal cartilage grafts. J Plast Reconstr Aesthet Surg 2008;61:1465-71. 